\title{
Pol-DinSAR: Polarimetric SAR Differential Interferometry Using Coherent Scatterers
}

\author{
Rafael Zandona Schneider and Konstantinos Papathanassiou \\ German Aerospace Center (DLR), Microwaves and Radar Institute \\ PO BOX 1116, 82230 Wessling, Germany
}

\begin{abstract}
We investigate the impact of polarimetry and polarimetric effects on the Differential Interferometry (DinSAR) technique for the coherent backscattering reciprocal case, with the aim to obtain better accuracy and to extract additional information about the scatterer. Emphasis is given to the effects of Faraday and scatterer Line-of-Sight (LOS) rotations, and to the determination of the intrinsic scatterer scattering mechanism.
\end{abstract}

\section{INTRODUCTION}

Differential Interferometry (DinSAR) is normally applied on spaceborne single polarization channel (HH or VV) SAR data. Examples of advanced DinSAR techniques make use of the so-called Permanent Scatterers (PSs), which give rise to great advantages when compared to standard DinSAR. Recently, the Coherent Scatterers (CSs) technique [1], which allows the detection of point-like scatterers with a reduced number of images, has been proposed. Such CSs are deterministic scatterers, characterized by high polarimetric coherence, and have DinSAR as potential application.

In this paper we study polarimetric effects for DinSAR purposes over such (polarimetric coherent) point-like scatterers. We investigate the use of fully polarimetric data in order to improve the estimations, as well as to obtain further information about the scatterer movement and intrinsic scattering mechanism.

\section{Scattering Matrix Symmetry Degrees}

In this section we propose an alternative parametrization (or decomposition) of the scattering matrix. The parametrization uses the CTBR (Consimilarity Transformation Based Routines) structure of the scattering matrix [2], and is based on certain degrees of symmetries of the corresponding scatterer.

Starting from the scattering matrix $S$, the CTBR LOS rotation angle $\psi$ is firstly removed [2]

$$
S_{u}=R^{T}(\psi) S R(\psi), \quad \text { with } \quad \psi=\frac{1}{2} \arctan \left\{\frac{\Re\left\{a c^{*}\right\}}{\Re\left\{a b^{*}\right\}}\right\}
$$

where $\Re\{x\}$ means the real part of $x, a, b$ and $c$ are the Pauli components $S=a S_{a}+b S_{b}+c S_{c}$, which can be evaluated by

$$
S=\left(\begin{array}{cc}
S_{H H} & S_{H V} \\
S_{V H} & S_{V V}
\end{array}\right)=\frac{1}{\sqrt{2}}\left(\begin{array}{cc}
a+b & c \\
c & a-b
\end{array}\right)
$$

if $S$ is given in the $H, V$ (Horizontal-Vertical) linear polarization basis. In this way, the underlying CTBR scatterer signature $S_{u}$ is obtained and is further parametrized as

$S_{u}=\left\|S_{u}\right\| e^{\jmath \phi_{a}}\left(\cos \alpha S_{a}+e^{\jmath \phi_{b a}} \sin \alpha \cos \delta S_{b}-\jmath \sin \alpha \sin \delta S_{c}\right)$

by projecting $S_{u}$ onto the Pauli basis. In the above equations, the Pauli basis $S_{a}, S_{b}, S_{c}$ and the rotation operator $R(\psi)$ read

$$
\begin{aligned}
& S_{a}=\frac{1}{\sqrt{2}}\left(\begin{array}{ll}
1 & 0 \\
0 & 1
\end{array}\right), \quad S_{b}=\frac{1}{\sqrt{2}}\left(\begin{array}{cc}
1 & 0 \\
0 & -1
\end{array}\right), \\
& S_{c}=\frac{1}{\sqrt{2}}\left(\begin{array}{ll}
0 & 1 \\
1 & 0
\end{array}\right), \quad R(\psi)=\left(\begin{array}{cc}
\cos \psi & -\sin \psi \\
\sin \psi & \cos \psi
\end{array}\right) .
\end{aligned}
$$

The $\alpha$ angle can be interpreted as the scatterer degree of rotation symmetry. When $\alpha=0$ the scatterer is totally rotation symmetric while $\alpha=\frac{\pi}{2}$ corresponds to totally rotation asymmetric scatterers. The angle $\delta$ can be seen as the scatterer degree of reflection symmetry (when used together with $\alpha$ ). A value of $\delta=0$ corresponds to a totally reflection symmetric scatterer while $\delta= \pm \frac{\pi}{2}$ (when $\alpha=\frac{\pi}{2}$ ) to a totally reflection asymmetric scatterer. The phase $\phi_{b a}$ is the phase between the second and third Pauli components of $S_{u}$ and can then be seen as a characteristic internal scatterer phase. It is necessary for the complete description of the polarimetric scatterer properties. Distinguishing reflection from rotation symmetry allows better insights on the physical nature of the underlying scatterer. Note that $\alpha$ in (3) is the same as the $\alpha$ angle defined in [3], since projections onto the first Pauli spin matrix $S_{a}$ are rotation independent, and hence the projection of either $S$ or $S_{u}$ onto $S_{a}$ are equivalent.

Fig. 1 presents the histograms of the previous defined scattering matrix parameters of Coherent Scatterers detected in the city of Munich in Germany, using L-band SAR data acquired by the E-SAR system of the German Aerospace Center (DLR) on October 2005. Fig. 1(a) shows the normalized histogram of the rotation symmetry degree $\alpha$, Fig. 1 (b) of the reflection symmetry degree $\delta$, and Fig. 1(c) of the phase $\phi_{b a}$. The majority of the CSs have rotation symmetry around $1.1 \mathrm{rad}$. There are not many CSs characterizes by total rotation symmetry with $\alpha=0$ or totally rotation asymmetric with $\alpha=\frac{\pi}{2}$. The majority of the CSs has a high reflection symmetry degree. However, a considerable amount of CSs has intermediary reflection symmetry, from zero up to $\pm \frac{\pi}{4}$. There are not too many occurrences of CSs having $|\delta|>\frac{\pi}{4}$. The angle $\phi_{b a}$ of CSs is well symmetrically distributed within $\left(-\frac{\pi}{2}, \frac{\pi}{2}\right]$, however with concentration around zero and with low occurrences around $\phi_{b a}= \pm \frac{\pi}{2}$. 


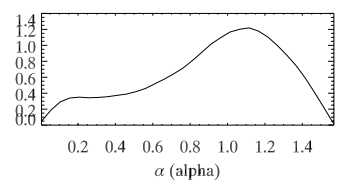

(a) Rotation symmetry $\alpha$

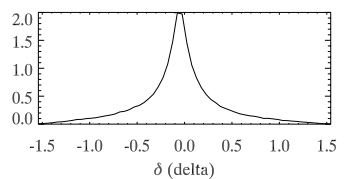

(b) Reflection symmetry $\delta$

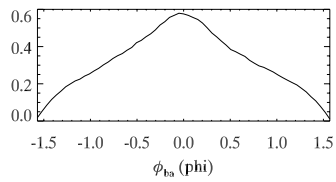

(c) Characteristic phase $\phi_{b a}$

Fig. 1. Histograms of the scattering matrix parameters.

\section{The EFFECT OF A LOS RotATION ON THE SCATTERING Matrix ElEMENTS}

Using the previous defined parameters, the terms of the scattering matrix of a (CTBR) non-rotated scatterer, expressend in the $\{\mathrm{H}-\mathrm{V}\}$ linear basis, are given by

$$
\begin{aligned}
& \bar{S}_{H H}=\left\|S_{u_{C}}\right\| e^{j \phi_{a}}\left(\cos \alpha+e^{j \phi_{b a}} \sin \alpha \cos \delta\right) \\
& \bar{S}_{V V}=\left\|S_{u_{C}}\right\| e^{j \phi_{a}}\left(\cos \alpha-e^{j \phi_{b a}} \sin \alpha \cos \delta\right) \\
& \bar{S}_{H V}=\left\|S_{u_{C}}\right\| e^{j \phi_{a}}(-j \sin \alpha \sin \delta)
\end{aligned}
$$

while when the scatterer is rotated by an angle $\psi$, the rotated scattering matrix terms read

$$
\begin{aligned}
S_{H H}= & \left\|S_{u_{C}}\right\| e^{j \phi_{a}}\left(\cos \alpha+e^{j \phi_{b a}} \sin \alpha \cos \delta \cos 2 \psi\right. \\
& +j \sin \alpha \sin \delta \sin 2 \psi) \\
S_{V V}= & \left\|S_{u_{C}}\right\| e^{j \phi_{a}}\left(\cos \alpha-e^{j \phi_{b a}} \sin \alpha \cos \delta \cos 2 \psi\right. \\
& -j \sin \alpha \sin \delta \sin 2 \psi) \\
S_{H V}= & \left\|S_{u_{C}}\right\| e^{j \phi_{a}}\left(e^{j \phi_{b a}} \sin \alpha \cos \delta \sin 2 \psi\right. \\
& -j \sin \alpha \sin \delta \cos 2 \psi) .
\end{aligned}
$$

Note that for reflection asymmetric scatterers $(\delta \neq 0)$, when $\psi \neq 0$ then the phase difference between the rotated and nonrotated $S$ matrix components is not zero: $\arg \left\{S_{m n} \bar{S}_{m n}^{*}\right\} \neq 0$ (for $m, n \in\{H, V\}$ ), when $\delta \neq 0$ and $\psi \neq 0$.

This means that a rotation about the LOS direction of a scatterer with reflection asymmetric characteristics induces a phase difference in the scattering matrix elements. This phenomenom will have implications in single polarization SAR differential interferometry, where the phase difference between acquisitions is the main input and is normally related to a displacement along the LOS direction. In such situations, the phase difference between the scattering matrix channels may be mis-interpreted, since the scatterer has rotated around and not moved along the LOS direction.

Additionally, observe from (5) and (6) that this effect may also happen even for symmetric scatterers $(\delta=0)$. Symmetric scatterers having $\phi_{b a} \neq 0$ also present phase variations in the scattering matrix elements when rotated around the LOS direction. Note that the only set of scatterers for which the rotation angle $\psi$ and the phases of the $S$ matrix terms are decoupled are the set of reflection symmetrical scatterers with no internal phase $\phi_{b a}$. For all other cases, absolute phase and rotation angle are coupled. Fig. 1 shows that a considerable number of CSs have $|\delta|>0$, and that for a large number $\left|\phi_{b a}\right|>0$. Hence, point-like scatterers in urban scenarios may be reflection asymmetric and have high internal phase differences.

In order to illustrate how large such phase variations may achieve, Figs. 2(a) to 2(c) shows the phases of the $S$ matrix terms $\left(\phi_{H H}, \phi_{H V}, \phi_{V H}\right.$ and $\left.\phi_{V V}\right)$ as a function of the rotation angle $\psi$ for three different scatterer types. Note that for all cases, the phases vary for different values of $\psi$. To convert the phases in displacement errors, one has to multiply them by the factor $\frac{\lambda}{4 \pi}$, which is dependent on the wavelength $\lambda$.

Such implications indicate that polarimetry and differential interferometry may require to be fused, in order to obtain more accurate results in certain situations.

\section{The EFfect of a Faraday Rotation on the SCATTERING MATRIX ElEMENTS}

Faraday rotation is the rotation of the electrical field vector of a polarized EM wave, when the wave travels through the ionosphere [4]. The ionosphere also affects the absolute phase of $S$, however this effect is not considered here. Faraday rotation in a radar backscatering configuration is modeled as

$$
\begin{aligned}
& S^{\prime}=\left[\begin{array}{cc}
S_{H H}^{\prime} & S_{H V}^{\prime} \\
S_{V H}^{\prime} & S_{V V}^{\prime}
\end{array}\right]=R^{T}(\Omega) S R^{T}(\Omega)= \\
& {\left[\begin{array}{cc}
\cos \Omega & \sin \Omega \\
-\sin \Omega & \cos \Omega
\end{array}\right]\left[\begin{array}{ll}
\bar{S}_{H H} & \bar{S}_{H V} \\
\bar{S}_{V H} & \bar{S}_{V V}
\end{array}\right]\left[\begin{array}{cc}
\cos \Omega & \sin \Omega \\
-\sin \Omega & \cos \Omega
\end{array}\right]}
\end{aligned}
$$

where $S^{\prime}$ is the rotated and $\bar{S}$ the non-rotated scattering matrix.

Using (5) and assuming reciprocity $\left(\bar{S}_{H V}=\bar{S}_{V H}\right)$ and no LOS rotation for the moment, one has for the $S^{\prime}$ matrix elements

$$
\begin{aligned}
S_{H H}^{\prime} & =\left\|S_{u_{C}}\right\| e^{j \phi_{a}}\left(\cos \alpha \cos 2 \Omega+e^{j \phi_{b a}} \sin \alpha \cos \delta\right) \\
S_{V V}^{\prime} & =\left\|S_{u_{C}}\right\| e^{j \phi_{a}}\left(\cos \alpha \cos 2 \Omega-e^{j \phi_{b a}} \sin \alpha \cos \delta\right) \\
S_{H V}^{\prime} & =\left\|S_{u_{C}}\right\| e^{j \phi_{a}}(-j \sin \alpha \sin \delta+\cos \alpha \sin 2 \Omega) \\
S_{V H}^{\prime} & =\left\|S_{u_{C}}\right\| e^{j \phi_{a}}(-j \sin \alpha \sin \delta-\cos \alpha \sin 2 \Omega) .
\end{aligned}
$$

Note from (8) and (5) that in general (for reflection asymmetric or even symmetric scatterers), Faraday rotation will also induce phase differences in the elements of the scattering matrix. From (8), the angle $\Omega$ for the coherent case can be estimated by [4]

$$
\Omega=\frac{1}{2} \arctan \left\{\frac{S_{H V}^{\prime}-S_{V H}^{\prime}}{S_{H H}^{\prime}+S_{V V}^{\prime}}\right\}
$$

Figs. 2(d) to 2(f) illustrate how large such phase variations may be for the same type of scatterers considered in the previous section. Note also in this case the coupling between Faraday rotation and the phases of the $S$ matrix terms. Reflection asymmetry will not influence the copolar channels, but the cross-polar, while rotation symmetry influences both. The internal characteristic phase also influences all the channel phases.

Also in this case, if single polarization DinSAR is performed in the presence of different Faraday rotations among acquisitions, incorrect measurements may be achieved when relating, directly, phase differences between scattering matrix 


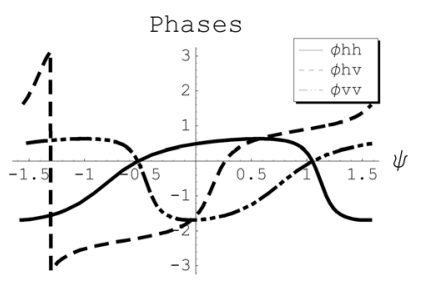

(a) $\alpha=\frac{\pi}{3}, \delta=\frac{\pi}{8}, \phi_{b a}=\frac{\pi}{4}$

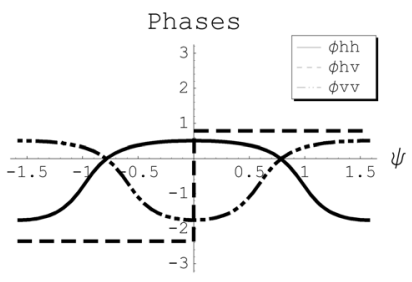

(c) $\alpha=\frac{\pi}{3}, \delta=0, \phi_{b a}=\frac{\pi}{4}$

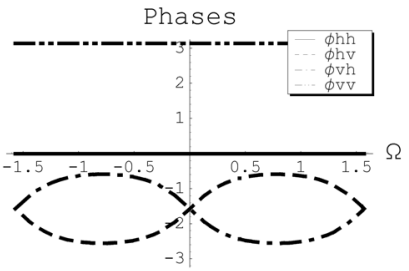

(e) $\alpha=\frac{\pi}{3}, \delta=\frac{\pi}{8}, \phi_{b a}=0$

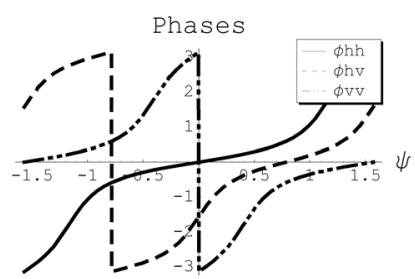

(b) $\alpha=\frac{\pi}{3}, \delta=\frac{\pi}{8}, \phi_{b a}=0$

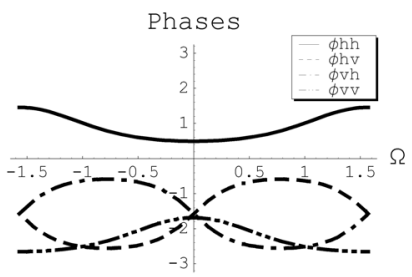

(d) $\alpha=\frac{\pi}{3}, \delta=\frac{\pi}{8}, \phi_{b a}=\frac{\pi}{4}$

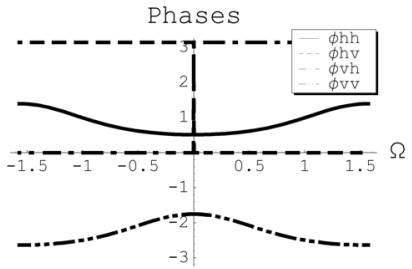

(f) $\alpha=\frac{\pi}{3}, \delta=0, \phi_{b a}=\frac{\pi}{4}$

Fig. 2. Scattering matrix phases for different scatterers, as function of scatterer $\psi$ and Faraday $\Omega$ rotations.

elements (co- or cross-polarization channels) to LOS displacements. This again indicates that for such situations polarimetry and DinSAR may need to be used together.

Note that the absolute phase of reflection symmetric scatterers with no internal phase, $\delta=0$ and $\phi_{b a}=0$, is not sensitive to Faraday as well as to the scatterer LOS rotation.

\section{POlarimetric DifFERENTIAL INTERFEROMETRY}

We adopt in this section a very simple DinSAR configuration, as the aim of the paper is to demonstrate polarimetric effects rather than precise DinSAR estimation techniques. Accordingly, we illustrate the effects on the three acquisitions DinSAR case, while extensions to multiple acquisitions are straightforward. It is assumed that the polarimetric scatterer signature $S_{u}$ remains the same among the acquisitions.

The scattering matrix for the first acquisition when no scatterer and Faraday rotation are present can be modelled as

$$
S_{1}=e^{j \frac{4 \pi}{\lambda} r_{1}} e^{j \varphi_{a p s_{1}}} e^{j \varphi_{N_{1}}} S_{u}
$$

and in a second acquisition, still assuming no rotations, as

$$
S_{2}=e^{j \frac{4 \pi}{\lambda} r_{2}} e^{j \varphi_{a p s_{2}}} e^{j \varphi_{N_{2}}} S_{u}
$$

while in the presence of both rotations and a scatterer movement along the LOS direction, in a third acquisition, one has

$S_{3}=e^{j \frac{4 \pi}{\lambda} r_{3}} e^{j \varphi_{m o v}} e^{j \varphi_{a p s_{3}}} e^{j \varphi_{N_{3}}} R^{T}(\Omega) R(\psi) S_{u} R^{T}(\psi) R^{T}(\Omega)$

with the corresponding absolute phases given by

$$
\begin{array}{ll}
\varphi_{1}=\frac{4 \pi}{\lambda} r_{1}+\varphi_{a p s_{1}}+\varphi_{N_{1}}+\varphi_{S_{1 p}} & =\frac{4 \pi}{\lambda} r_{1}+\varphi_{e_{1}} \\
\varphi_{2}=\frac{4 \pi}{\lambda} r_{2}+\varphi_{a p s_{2}}+\varphi_{N_{2}}+\varphi_{S_{2 p}} & =\frac{4 \pi}{\lambda} r_{2}+\varphi_{e_{2}} \\
\varphi_{3}=\frac{4 \pi}{\lambda} r_{3}+\varphi_{m o v}+\varphi_{a p s_{3}}+\varphi_{N_{3}}+\varphi_{S_{3 p}} & =\frac{4 \pi}{\lambda} r_{3}+\varphi_{e_{3}}
\end{array}
$$

where $r_{i}$ are the distances radar-scatterer, $\varphi_{a p s_{i}}$ are the phases induced by the atmospheric phase screen, $\varphi_{\text {mov }}$ is the phase induced by the scatterer movement, $\varphi_{N_{i}}$ are additional noise contributions, and $\varphi_{S_{i p}}$ for $i=\{1,2,3\}$ are the absolute phases of the scattering matrices $S_{i}$, where $p$ stands for the polarimetric channel (transmit and receive polarizations), e.g., $p=H H, V V, H V$, etc. . Note that $\varphi_{a p s_{i}}$ and $\varphi_{N_{i}}$ may be also polarimetric dependent, although not assumed here.

The interferograms $\phi_{21}=\varphi_{2}-\varphi_{1}$ and $\phi_{31}=\varphi_{3}-\varphi_{1}$ read

$$
\begin{aligned}
& \phi_{21}=\frac{4 \pi}{\lambda}\left(r_{2}-r_{1}\right)+\left(\varphi_{e_{2}}-\varphi_{e_{1}}\right) \simeq \kappa_{z_{1}} h+\left(\varphi_{e_{2}}-\varphi_{e_{1}}\right) \\
& \phi_{31}=\frac{4 \pi}{\lambda}\left(r_{3}-r_{1}\right)+\left(\varphi_{e_{3}}-\varphi_{e_{1}}\right) \simeq \kappa_{z_{2}} h+\left(\varphi_{e_{3}}-\varphi_{e_{1}}\right)
\end{aligned}
$$

where $\kappa_{z_{i}}$ are the vertical wave numbers and $h$ is the scatterer original (first two acquisitions) height.

The differential interferogram with the projection of the first onto the second interferometric pair geometry $\Delta \phi=\phi_{31}-$ $\frac{\kappa_{z_{2}}}{\kappa_{z_{1}}} \phi_{21}$ is then given by

$$
\begin{aligned}
\Delta \phi= & \left(\varphi_{e_{3}}-\varphi_{e_{1}}\right)-\frac{\kappa_{z_{2}}}{\kappa_{z_{1}}}\left(\varphi_{e_{2}}-\varphi_{e_{1}}\right) \\
= & \varphi_{m_{o v}}+\left(\phi_{a p s_{31}}\right)+\left(\phi_{N_{31}}\right)+\left(\varphi_{S_{3 p}}-\varphi_{S_{1 p}}\right) \\
& +\frac{\kappa_{z_{2}}}{\kappa_{z_{1}}}\left[\left(\phi_{a p s_{21}}\right)+\left(\phi_{N_{21}}\right)+\left(\varphi_{S_{2 p}}-\varphi_{S_{1 p}}\right)\right]
\end{aligned}
$$

where $\phi_{a p s_{j 1}}=\varphi_{a p s_{j}}-\varphi_{a p s_{1}}$ and $\phi_{N_{j 1}}=\varphi_{N_{j}}-\varphi_{N_{1}}$ for $j=\{2,3\}$. As we have assumed for simplicity that no Faraday and scatterer LOS rotations were present between the two first acquisitions and that $S_{u}$ reminds constant, $\varphi_{S_{2 p}}-\varphi_{S_{1 p}}=0$. We arrived then to the standard DinSAR configuration, up to the differential phase term $\phi_{S_{31 p}}=\varphi_{S_{3 p}}-\varphi_{S_{1 p}}$, which is dependent on the Faraday rotation and scatterer LOS rotation present in the third acquisition, as well as on the scatterer type (parameters, $\alpha, \delta, \phi_{b a}$ ), if any rotation is present.

For the $H H$ channel, for example, $\phi_{S_{31 H}}$ reads

$$
\begin{aligned}
\phi_{S_{31 H H}}= & \arg \left\{\cos \alpha \cos \Omega+e^{j \phi_{b a}} \sin \alpha \cos \delta \cos 2 \psi\right. \\
& +j \sin \alpha \sin \delta \sin 2 \psi\}-\arg \{\cos \alpha+ \\
& \left.e^{j \phi_{b a}} \sin \alpha \cos \delta\right\}
\end{aligned}
$$

Hence, it is clear that Faraday rotation as well as a scatterer LOS rotation will induce, in the general case, additional phase differences in single polarization DinSAR. If full polarimetric data are available, such effects can be compensated using (1) and (3) to estimate $\alpha, \delta, \phi_{b a}, \psi, \Omega$ and hence $\phi_{S_{31 p}}$. Note that by estimating these parameters, not only $\phi_{S_{31 p}}$ can be compensated but also further information about the scatterer position $(\psi)$, ionospheric activity $(\Omega)$, as well as the intrinsic scatterer scattering mechanism $\left(\alpha, \delta, \phi_{b a}\right)$ could be obtained. After the compensation of $\phi_{S_{31 p}}$, standard DinSAR techniques may be applied for the estimation of the scatterer LOS displacement $\left(\varphi_{\text {mov }}\right)$ and atmospheric artefacts $\phi_{a p s}$. 


\section{The Absolute Phase of the Scattering Matrix}

The absolute phase (AP) of the scattering matrix is arbitrary. In fact, there is an infinite number of APs of $S$ as there is an infinite number of combinations of transmit and receive polarization states of an EM wave. Sometimes it is necessary to establish a reference by choosing one of them. In the majority of the cases, there is a dependence (coupling) between the LOS rotation angle of the scatterer and the APs of the corresponding $S$ matrix.

Huynen defined the AP $\rho$ of the $S$ matrix after the consimilarity transformation $S^{\prime}=U^{T}(\psi, \tau) S U(\psi, \tau)=S_{d}=$ $\operatorname{diag}\left[\lambda_{1}, \lambda_{2}\right]$, where $U(\psi, \tau)$ is a unitary matrix and $\tau$ is the scatterer ellipsity, as

$$
S_{d}=\left(\begin{array}{cc}
\lambda_{1} & 0 \\
0 & \lambda_{2}
\end{array}\right)=m e^{j \rho}\left(\begin{array}{cc}
e^{j 2 \nu} & 0 \\
0 & \tan ^{2} \gamma e^{-j 2 \nu}
\end{array}\right)
$$

with $0 \leq \gamma \leq \pi / 4$ and $-\pi / 4 \leq \nu \leq \pi / 4$, where $\lambda_{i}$ are the coneigenvalues of the $S$ matrix. Krogager proposed one alternative definition to circumvent undeterminations for the cases in which one coneigenvalue is zero, by defining the reference AP as the phase of the first coneigenvalue $\lambda_{1}$, i.e., $\xi=\rho+2 \nu$. The Krogager definition seems to have advantages in the sense that one coneigenvalue always exist. It has problems when the amplitude of both coneigenvalues are equal, but such are cases for which the consimilarity transformation can not be applied, and hence, special cases.

Both definitions are rotation independent since the rotation angle is removed through the consimilarity transformation. The same could be done for the AP of the $S_{H H}$ channel by saying: first estimate the rotation angle $\psi$ (and the other parameters) and then remove its influence from the $S_{H H}$ channel using (6). This way the phase of the 'compensated $S_{H H}$ ' becomes also rotation independent. Note that to perform the rotation angle estimation as well as the whole consimilarity transformation, the compensation of $\Omega$ has to be performed first, using (9).

However, if one is not interested in the estimation of $\psi$ and $\Omega$ as well as of $\alpha, \delta, \phi_{b a}$, but just wants to get rid of the Faraday and scatterer LOS rotation effects, the definition in (3) of $\phi_{a}$ has advantages in being used as the reference absolute phase of the $S$ matrix, and in its utilization for DinSAR purposes. The phase $\phi_{a}$ is the phase associated to the rotation symmetry degree, which by definition is rotation independent, being decoupled from $\psi$. Furthermore, after a Faraday rotation, the rotation symmetry and its associated phase become

$$
e^{j \phi_{a}^{\prime}} \cos \alpha^{\prime}=e^{j \phi_{a}} \cos \alpha \cos 2 \Omega
$$

what indicates that only its amplitude is changed but not the phase $\phi_{a}$, for values of $\Omega$ in $\left(-\frac{\pi}{4}, \frac{\pi}{4}\right]$. For $\Omega \in\left(-\frac{\pi}{2},-\frac{\pi}{4}\right]$ or $\Omega \in\left(\frac{\pi}{4}, \frac{\pi}{2}\right]$ there will be $\pm \pi$ discontinuities in $\phi_{a}^{\prime}$, what should in general not be difficult to identify.

The phase $\phi_{a}^{\prime}$, which is directly estimated by

$$
\phi_{a}^{\prime}=\arg \left\{S_{H H}^{\prime}+S_{V V}^{\prime}\right\}
$$

and hence with no need to perform the consimilarity transformation, is not only insensitive to a scatterer LOS rotation,

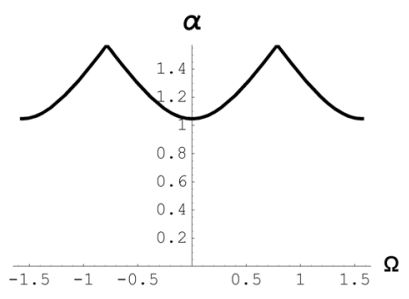

(a) $\alpha$ under Faraday rotation

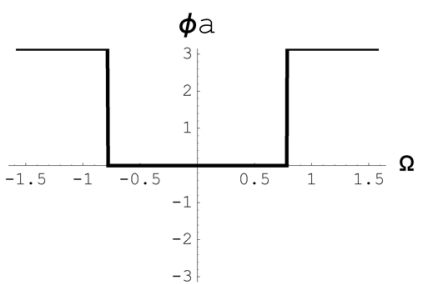

(b) $\phi_{a}$ under Faraday rotation
Fig. 3. $\alpha$ and $\phi_{a}$ under Faraday rotation $\Omega$

but also to a Faraday rotation (in the above interval), for all type of scatterers, (off course with the exception of scatterers having $\alpha=\frac{\pi}{2}$, for which it is not defined).

The only disadvantage of such reference AP is that it main become too sensitive to noise for high values of $\alpha$. A reference AP less sensitive to noise is the one defined by Krogager because it is the phase of the polarization state leading to maximum returned power; but it requires the evaluation of the consimilarity transformation, being a 'scatterer dependent AP' rather than a fix AP as $S_{H H}$ or $\phi_{a}$. Finally, from (6) and (8) follows that the phase of the second Pauli component $b$ (ideal dihedral) is insensitive to Faraday rotation but is sensitive to the scatterer LOS rotation, for general scatterer types.

Fig. 3 shows $\alpha^{\prime}$ and the phase $\phi_{a}^{\prime}$ for the cases of Figs. 2(d) to 2(f). Note that while $\alpha^{\prime}$ changes with $\Omega$, the phase $\phi_{a}^{\prime}$ remains constant for $\Omega$ in $\left(-\frac{\pi}{4}, \frac{\pi}{4}\right]$, but has a jump of $\pi$ for $\Omega<-\frac{\pi}{4}$ or $\Omega>\frac{\pi}{4}$. For the cases of Figs. 2(a) to 2(c) $\phi_{a}^{\prime}=\phi_{a}=$ cte.

\section{CONCLUSIONS}

Polarimetric effects and their influence on DinSAR techniques have been addressed in the paper. Using the proposed symmetry degrees we demonstrated that many CSs in urban areas are reflection asymmetric and/or have high internal characteristic phases. For these scatterers, in the presence of Faraday or a scatterer LOS rotation, implications leading to mis-interpretation of the DinSAR phase may arise.

Polarimetry not only allows the correction of such effects, but also the estimation of the Faraday and scatterer LOS and rotation, as well as additional information about the scatterer scattering mechanism. If one is not interested on such extra information, a quick and direct absolute phase $\left(\phi_{a}\right)$ estimation may be used for DinSAR purposes, which, as shown, is insensitive to scatterer as well as to Faraday rotation.

\section{REFERENCES}

[1] R. Z. Schneider, K. P. Papathanassiou, I. Hajnsek, A. Moreira, Polarimetric and Interferometric Characterization of Coherent Scatterers in Urban Areas, IEEE Trans. Geoscience and Remote Sensing, vol. 44, No. 4, pp. 971-984, April 2006

[2] R. Z. Schneider and K. P. Papathanassiou, A Study on LOS Rotation Angle Estimation Procedures in RADAR Polarimetry, IEEE Trans. Geoscience and Remote Sensing, to be submitted.

[3] S. R. Cloude and E. Pottier, An Entropy based Classification Scheme for Land Applications of Polarimetric SAR, IEEE Trans. Geoscience and Remote Sensing, vol. 35, no. 1, pp. 68-78, January 1997.

[4] A. Freeman and S. S. Saatchi, The Effects of Faraday Rotation on Backscatter Signatures in SAR Image Data, JPL TRS, January 1997. Available on http://hdl.handle.net/2014/23038 